\title{
FERTILIZAÇÃO DE PLANTAS ORNAMENTAIS PELO MÉTODO REQUERIMENTO-SUPRIMENTO: PROPOSIÇÃO DE TÉCNICA EXPERIMENTAL ${ }^{(1)}$
}

\author{
Víctor Hugo Alvarez V.(2), André Ferreira Santos ${ }^{(3)}$, Gláucio Leboso Alemparte Abrantes \\ dos Santos ${ }^{(4)} \&$ Patrícia Morais da Matta ${ }^{(5)}$
}

\section{RESUMO}

A dose de fertilizantes a ser recomendada para uma cultura depende do balanço de perdas e dos ganhos dos nutrientes no sistema agrícola (Lei da Restituição). Na UFV, o balanço foi modelado para recomendação de corretivos e fertilizantes para eucalipto (NUTRICALC), abacaxizeiro, algodoeiro, arroz, bananeira, cafeeiro, cana de açúcar, coqueiro, laranjeira, meloeiro, milho, pastagens, soja e tomate (FERTICALC) e teca (FERTI-UFV). A fertilização e a nutrição de plantas ornamentais tropicais baseam-se apenas na experiência de produtores e fabricantes de fertilizantes. Os objetivos deste trabalho foram desenvolver e apresentar técnica experimental que permita determinar: as taxas de recuperação de macro e micronutrientes por extratores de formas disponíveis, a demanda e as taxas de recuperação de nutrientes por plantas ornamentais e determinar a dose e os teores de nutrientes em fertilizante que suplementem os requerimentos das plantas. Para a modelagem, consideraram-se dois módulos: o módulo planta, que obtém a demanda e o requerimento dos nutrientes para definida produtividade; $e$ o módulo substrato, que permite calcular o suprimento de nutrientes do vaso ou do canteiro a ser utilizado para o cultivo. A modelagem vislumbrou um novo método de pesquisa para recomendação de corretivos e fertilizantes para culturas com carência de informação, como para cultivo de plantas ornamentais, especialmente as tropicais. Esse Método Requerimento-Suprimento determina a dose e os teores de nutrientes no fertilizante-suprimento (FS), que suplementam os requerimentos das plantas. $\mathrm{O}$ método é iterativo e foi desenvolvido por meio de três tentativas. $\mathrm{Na}$ primeira tentativa, considerou-se a produção de plântulas de orquídea; e, na segunda e terceira tentativas, determinaram-se as curvas de crescimento com FS no cultivo de violetas, em vasos com substrato, e definiram-se os critérios para determinar as

\footnotetext{
(1) Recebido para publicação 11 de abril de 2013 e aprovado em 6 de janeiro de 2014.

(2) Professor Voluntário do Departamento de Solos - DPS, Universidade Federal de Viçosa - UFV. Av. P. H. Rolfs, s/n. CEP 36570900 Viçosa (MG). Bolsista do CNPq. E-mail: vhav@ufv.br

(3) Doutorando, DPS/UFV. E-mail: s.andreferreira@gmail.com

(4) Professor Substituto, Universidade Estadual de Mato Grosso do Sul, Cidade Universitária de Dourados. Caixa Postal 351. CEP 79804-970 Dourados (MS). E-mail: glaucio@agronomo.eng.br

(5) Doutoranda, DPS/UFV. E-mail: matta542@gmail.com
} 
taxas de recuperação de nutrientes pelos extratores em análises químicas do substrato e pelas plantas. Por último, apresentou-se a técnica experimental projetada. Termos de indexação: técnica experimental, demanda de nutrientes,
recomendação de fertilizantes.

\title{
SUMMARY: FERTILIZATION OF ORNAMENTAL PLANTS: REQUIREMENT- SUPPLY METHOD
}

\begin{abstract}
The recommendation of a fertilizer dose for a crop depends on nutrient gain-loss balance in the agricultural system (Restitution Law). At the Federal University of Viçosa (UFV), this balance has been modeled for liming and fertilizer recommendations for eucalyptus (NUTRICALC), pineapple, cotton, rice, banana, coffee, sugarcane, coconut, orange, melon, maize, pastures, soybean, tomato (FERTICALC), and for teak (FERTI - UFV). Fertilization and nutrition of tropical ornamental plants is based on the knowledge of growers and fertilizer manufacturers. This study was developed to establish an experimental technique for determining: recovery rates of available forms of macro and micronutrients by extractors; nutrient demand and recovery rates of ornamental plants; as well as nutrient doses and contents in fertilizer which supplies plant requirements. The modeling focused on two modules. Plant module, in which nutrient demand and requirement for a given yield are obtained; and substrate module, that allows to calculate nutrient supply from the pot or bed used for cultivation. Modeling enabled to outline a new research method for lime and fertilizer recommendations of crops with insufficient information, particularly for tropical ornamental plants. The requirement - supply method allows to determine nutrient dose and content of supply fertilizer (SF) that meets plant requirement. The method is iterative and was developed in three approaches. The first one considered the production of orchid seedlings. In the second, growth curves with SF were determined for violets cultivated in pots containing substrate. In the last, criteria to define nutrient recovery rates in plants and substrate chemical extraction were determined. Finally, the proposed experimental technique is presented.
\end{abstract}

Index terms: experimental technique, nutrient demand, fertilizer recommendation.

\section{INTRODUÇÃO}

A fertilização e a nutrição de plantas ornamentais baseam-se apenas na experiência de produtores e fabricantes de fertilizantes, o que resulta em indicações de doses de fertilizantes frequentemente controversas.

A prática da fertilização de uma cultura tem sido definida como: que fertilizante; em que dose e em que época deve ser adicionada; e para que, em determinado talhão, as plantas disponham dos nutrientes em quantidades suficientes e em proporções adequadas (Merle, 1959).

Em relação à dose de um nutriente, devem-se conhecer, ao mesmo tempo, a demanda das plantas ao longo do seu desenvolvimento e crescimento vegetativo e produtivo, o coeficiente de utilização do nutriente e, também, o teor do nutriente do solo com seu coeficiente de utilização e sua velocidade de transporte (Merle, 1959).

Fica evidente que a dose a ser recomendada depende do balanço de perdas e ganhos dos nutrientes no sistema agrícola, ou seja, da aplicação da Lei da Restituição.
Essas ideias têm constituído a base conceitual que nortearam, pelas últimas duas décadas, os trabalhos de modelagem do grupo de professores do Departamento de Solos da Universidade Federal de Viçosa para recomendação de corretivos e fertilizantes para eucalipto, NUTRICALC (Barros et al., 1995), e várias outras culturas como FERTICALC (Novais \& Smyth, 1999); abacaxizeiro, algodoeiro, arroz, bananeira, cafeeiro arábica, cana de açúcar, coqueiro, milho, pastagens, soja, tomate, meloeiro (Deus, 2012); laranjeira (Stahringer, 2013); e teca FERTI-UFV Teca (Pontes, 2011).

Para a modelagem, consideraram-se dois módulos: o módulo planta, que obtém a demanda e o requerimento dos nutrientes para definida produtividade; e o módulo solo, que permite calcular o suprimento de nutrientes do talhão a ser utilizado para o cultivo.

A modelagem constatou a necessidade de grande volume de informação, detectou os vazios de informação existentes, bem como vislumbrou novo método de pesquisa para recomendação de corretivos e fertilizantes para culturas com carência de informação, como para cultivo de plantas ornamentais, especialmente as tropicais. 
Para utilização desses conceitos e métodos, dentro de nova técnica experimental a ser utilizada para recomendação da dose e dos teores de nutrientes no fertilizante, na produção de plantas ornamentais, é necessário considerar que: a demanda de nutrientes será alcançada facilmente da análise de amostras de plantas de qualidade superior, obtidas no comércio; o requerimento pode ser estimado com base na demanda e na utilização, inicialmente, de taxas de recuperação extrapoladas de plantas de outras culturas; e o suprimento será obtido para sistemas mais homogêneos que os solos, pois se trabalhará com substratos corrigidos. Portanto, a recomendação de fertilizantes será para fertilização de manutenção.

Os objetivos deste trabalho foram desenvolver e apresentar técnica experimental que permita determinar: as taxas de recuperação de macro e micronutrientes pelos extratores de formas disponíveis; a demanda e as taxas de recuperação de nutrientes por plantas ornamentais; e a dose e os teores de nutrientes no fertilizante-suprimento (FS) que suplementem os requerimentos das plantas.

\section{Marco conceitual}

$\mathrm{O}$ requerimento de nutrientes $\left(r \mathrm{Nu}_{\mathrm{i}}\right)$ é a demanda dos nutrientes $\left(d \mathrm{Nu}_{\mathrm{i}}\right)$ pela planta, corrigida pela taxa de recuperação desses pela planta $\left(t r \mathrm{Nu}_{\mathrm{i}}-\mathrm{Pl}\right)$ : $r \mathrm{Nu}_{\mathrm{i}}=d \mathrm{Nu}_{\mathrm{i}} / t r \mathrm{Nu}_{\mathrm{i}}-\mathrm{Pl}$

A demanda é determinada em amostra de plantas de qualidade comercial superior. É o somatório dos conteúdos dos nutrientes i nos diferentes compartimentos j das plantas $\left(c \mathrm{Nu}_{\mathrm{ij}}\right): d \mathrm{Nu}_{\mathrm{i}}=\Sigma_{1}{ }^{\mathrm{j}} c \mathrm{Nu}_{\mathrm{ij}}$.

$\mathrm{O} c \mathrm{Nu}_{\mathrm{ij}}$ é determinado com base na massa de matéria seca $\left(m \mathrm{MS}_{\mathrm{j}}\right)$ e no teor total do nutriente $\mathrm{i}$ por compartimento j das plantas $\left(t t \mathrm{Nu}_{\mathrm{ij}}\right)$ : $c \mathrm{Nu}_{\mathrm{ij}}=m \mathrm{MS}_{\mathrm{j}} \times t t \mathrm{Nu}_{\mathrm{ij}}$

A $t r \mathrm{Nu}_{\mathrm{i}}-\mathrm{Pl}$ depende da dinâmica dos nutrientes no contínuo substrato-solução-planta (inclusive da dose aplicada) e da eficiência de absorção e utilização desses pelas plantas.

Para as plantas ornamentais, o suprimento dos nutrientes $\left(s \mathrm{Nu}_{\mathrm{i}}\right)$ é determinado em amostras do substrato de cultivo, com base no teor médio de formas disponíveis $\left(t d \mathrm{Nu}_{\mathrm{i}}-\mathrm{Sb}\right)$ e na taxa de recuperação dos nutrientes pelo extrator $\left(t r \mathrm{Nu}_{\mathrm{i}}-\mathrm{Ex}\right)$ : $s \mathrm{Nu}_{\mathrm{i}}=t d \mathrm{Nu}_{\mathrm{i}}-\mathrm{Sb} / t r \mathrm{Nu}_{\mathrm{i}}-\mathrm{Ex}$.

O método Requerimento-Suprimento é iterativo, pois as taxas $t r \mathrm{Nu}_{\mathrm{i}}$ - Ex e $t r \mathrm{Nu}_{\mathrm{i}}-\mathrm{Pl}$, inicialmente, são desconhecidas para substratos e plantas ornamentais. $\mathrm{Na}$ primeira aproximação, serão utilizadas taxas encontradas na literatura para outros substratos e para outras culturas.

\section{Desenvolvimento do método}

\section{Primeira tentativa: cultivo de explantes de orquídeas em meio de cultura}

Para essa tentativa, considerou-se situação bem simples: a produção de plântulas de orquídea a partir de explantes (Santos, 2008). Para determinar as doses de nutrientes que serão adicionadas ao meio de cultura, o conteúdo de nutrientes foi definido em amostras das plântulas de frascos, analisadas como um todo. Também, considerou-se que o suprimento de nutrientes pelo meio de cultura seria nulo. Portanto, o requerimento indicaria as doses de nutrientes a serem adicionadas ao meio de cultura.

Inicialmente, para se estabelecer a formulação dos nutrientes no meio de cultura, foram considerados os teores totais de nutrientes nos tecidos de plântulas $\left(t t \mathrm{Nu}_{\mathrm{i}}\right)$ de Cattleya livres de sintomas de deficiências nutricionais e com elevada produção de matéria fresca. As concentrações dos nutrientes para o meio de cultura (Fertilizante-Suprimento - FS1) foram estabelecidas, considerando-se a produção de matéria seca esperada ( $0,7 \mathrm{~g} / \mathrm{frasco})$, as taxas de recuperação dos nutrientes pelas plântulas e o volume $(40 \mathrm{~mL})$ do meio de cultura utilizado por frasco (Quadro 1). Para exemplo, indicaram-se unicamente N, P, S, K, Ca, B, Zn e Fe e excluíram-se Mg, Mo, Mn e Cu.

Esse meio de cultura foi testado em quatro doses $\left(\mathrm{FS}_{0,55}, \mathrm{FS}_{0,75}, \mathrm{FS}_{1,00}\right.$ e $\left.\mathrm{FS} 1_{1,45}\right)$ e comparado com outros meios. Para este estudo, foi conduzido um experimento por $190 \mathrm{~d}$, utilizando 20 protocormos recém-geminados de Cattleya walkeriana var. coerulea $\times$ self, por frasco.

O meio de cultura com o fertilizante FS1, na dose de máxima produção, foi superior aos outros meios, com os quais foi comparado (Santos, 2008).

A produção máxima de MS de plântulas $(0,774 \mathrm{~g} /$ frasco) foi obtida com $F S 1_{1,125}$. Para maior produção de matéria seca de plântulas de orquídea ( $0,9 \mathrm{~g} /$ frasco), foi estimada a dose de novo FS (FS2) com os teores dos nutrientes em proporção específica e aproximadas novas $t r \mathrm{Nu}_{\mathrm{i}} \mathrm{Pl}$ (Quadro 2).

Observam-se as diferencias nas doses $\left(r \mathrm{Nu}_{\mathrm{i}}\right)$ e nas taxas $\left(t r \mathrm{Nu}_{\mathrm{i}}-\mathrm{Pl}\right)$ entre as duas aproximações (Quadros 1 e 2 ).

Essa técnica permitiu determinar o $r \mathrm{Nu}_{\mathrm{i}}$ e obter a recomendação das doses de nutrientes no FS2, sendo recomendada a dose de FS2 de 158,8 mg/frasco no meio de cultura para orquídea, mantendo no FS2 os teores de, em \% (m/m), : 10,00 N; 4,96 P; 3,14 S; $16,70 \mathrm{~K} ; 7,56 \mathrm{Ca} ; 1,89 \mathrm{Mg} ; 0,0708 \mathrm{~B} ; 0,0014 \mathrm{Mo}$; 0,227 Fe; 0,063 Zn; 0,090 Mn; e 0,0071 Cu.

\section{Segunda tentativa: Cultivo de violetas em substrato}

A segunda tentativa foi realizada para determinar curvas de crescimento de plantas de violeta africana (Saintpaulia ionantha Wendl) cultivadas em vasos com substrato, em casa de vegetação (Santos, 2011).

O fertilizante suprimento (FS1) foi determinado, inicialmente, com base na análise de cinco plantas em plena floração adquiridas no comércio.

A unidade experimental (UE) utilizada foi constituída por três vasos com $0,4 \mathrm{dm}^{3}$ de substrato e uma planta. 
Neste estudo, foram definidos a produção de matéria seca $(m \mathrm{MS})$, os teores totais $\left(t t \mathrm{Nu}_{\mathrm{i}}\right)$ e os conteúdos $\left(c \mathrm{Nu}_{\mathrm{i}}\right)$ de macro e micronutrientes de plantas de três variedades de violeta (Kalise de flores rosa, Krs; Kalise de flores vermelhas, Kvr; e Fiesta de flores branco e azul, Fba), cultivadas com duas doses de FS1, meia e duas vezes a dose recomendada do fertilizante suprimento (FS1) $\left[62,5\right.$ e $250 \mathrm{mg} \mathrm{dm}^{-3}$ por aplicação(aplic)], durante oito épocas distintas de colheita com intervalo de três semanas. Dessa maneira, houve um fatorial $3 \times 2 \times 8$. Os fertilizantes foram aplicados a cada 10 e $11 \mathrm{~d}$, e a cada $21 \mathrm{~d}$ as plantas da UE eram colhidas.

No momento da chegada das mudas, realizou-se a primeira coleta, correspondente à época $0 \mathrm{~d}$. Nas coletas, as plantas foram retiradas dos vasos, lavadas e divididas em raízes, região meristemática, folhas e inflorescências. Os órgãos vegetais j coletados foram secos em estufa de circulação forçada de ar a $70{ }^{\circ} \mathrm{C}$ para obtenção da $m \mathrm{MS}_{\mathrm{j}}$, moídos e submetidos à digestão nítrico-perclórica para a determinação dos teores totais de N, P, S, K, Ca, Mg, B, Mo, Fe, Zn, Mn e Cu. Os teores de P, S, K, Ca, Mg, B, Mo, Fe, Zn, Mn e $\mathrm{Cu}$ foram determinados por espectrometria de emissão ótica em plasma induzido (ICP-OES), e o teor de $\mathrm{N}$ foi determinado pelo método Kjeldahl.

A partir do teor total dos nutrientes $\left(t t \mathrm{Nu}_{\mathrm{ij}}\right)$ e da massa de matéria seca de cada órgão vegetal $\left(m \mathrm{MS}_{\mathrm{j}}\right)$ da planta, foi obtido o conteúdo do nutriente por órgão vegetal $c \mathrm{Nu}_{\mathrm{ij}}=m \mathrm{MS}_{\mathrm{j}} \times t t \mathrm{Nu}_{\mathrm{ij}}$; pelo somatório dos conteúdos nas raízes, no meristema, nas folhas e nas inflorescências, obteve-se a demanda de nutrientes pela planta: $d \mathrm{Nu}_{\mathrm{i}}=\Sigma_{1}{ }^{\mathrm{j}} c \mathrm{Nu}_{\mathrm{ij}}$.

Para as duas doses de FS1, ajustaram-se as equações de regressões da produção de matéria seca e dos conteúdos de nutrientes em função do tempo, para que fossem determinadas as demandas dos nutrientes ao longo do crescimento das plantas de violeta.

Por meio de contrastes, verificou-se que a variedade Krs produziu mais matéria seca por planta que Kvr e que Fba produziu menos matéria seca por planta que Krs e Kvr. Essa resposta repetiu-se para conteúdo de N, P, S, K, Ca, Mg, B, Mo e Fe. A dose $62,5 \mathrm{mg} / \mathrm{dm}^{3} /$ aplic de FS1 proporcionou maior produção de matéria seca por planta que a de $250 \mathrm{mg} / \mathrm{dm}^{3} /$ aplic em Fba e Krs (e na variedade Kvr, porém não significativamente), evidenciando que a dose menor foi adequada para proporcionar bom crescimento das plantas e a maior, excessiva. Consideraram-se apenas as curvas de demanda da variedade $\mathrm{Krs}$, na dose $62,5 \mathrm{mg} / \mathrm{dm}^{3} /$ aplic, para estimar, por época, as demandas dos nutrientes (Quadro 3).

Em cada época, pela divisão da demanda nutricional ( $d \mathrm{Nu}_{i}$, gerada pelos modelos de regressões) e pela taxa de recuperação do nutriente pela planta $\left(t r \mathrm{Nu}_{\mathrm{i}}-\mathrm{Pl}\right)$, chegou-se ao requerimento do nutriente: $r \mathrm{Nu}_{\mathrm{i}}=d \mathrm{Nu}_{\mathrm{i}} / t r \mathrm{Nu}_{\mathrm{i}}-\mathrm{Pl}$.

A $t r \mathrm{Nu}_{\mathrm{i}}-\mathrm{Pl}$ foi estimada pela divisão da demanda $\left(d \mathrm{Nu}_{\mathrm{i}}\right)$ e pelo suprimento do nutriente $\left(\mathrm{sNu}_{\mathrm{i}}\right): \operatorname{tr} \mathrm{Nu}_{\mathrm{i}}{ }^{-}$ $\mathrm{Pl}=d \mathrm{Nu}_{\mathrm{i}} / s \mathrm{Nu}_{\mathrm{i}}$.

Quadro 1. Teores e demanda pelas plântulas de orquídea e requerimento de nutrientes a serem adicionados no meio de cultura

\begin{tabular}{|c|c|c|c|c|c|c|c|c|c|}
\hline Variável & Unidade & $\mathbf{N}$ & $\mathbf{P}$ & $\mathbf{S}$ & $\mathbf{K}$ & $\mathbf{C a}$ & B & $\mathrm{Zn}$ & $\mathbf{F e}$ \\
\hline$t t \mathrm{Nu}_{\mathrm{i}}$ & $\mathrm{g} \mathrm{kg}^{-1}$ & 15,000 & 6,000 & 3,860 & 28,000 & 9,000 & 0,100 & 0,080 & 0,150 \\
\hline$d \mathrm{Nu}_{\mathrm{i}}^{(1)}$ & $\mathrm{mg} /$ frasco & 10,500 & 4,200 & 2,702 & 19,600 & 6,300 & 0,070 & 0,056 & 0,105 \\
\hline $\operatorname{tr} \mathrm{Nu}_{\mathrm{i}}-\mathrm{Pl}$ & & 0,800 & 0,700 & 0,600 & 0,700 & 0,800 & 0,800 & 0,700 & 0,400 \\
\hline$r \mathrm{Nu}_{\mathrm{i}}^{(2)}$ & $\mathrm{mg} /$ frasco & 13,125 & 6,000 & 4,503 & 28,000 & 7,875 & 0,088 & 0,080 & 0,263 \\
\hline$r \mathrm{Nu}_{\mathrm{i}}^{(3)}$ & $\mathrm{mg} \mathrm{L}^{-1}$ & 328,125 & 150,000 & 112,583 & 700,000 & 196,875 & 2,188 & 2,000 & 6,563 \\
\hline
\end{tabular}

(1) Demanda de nutrientes para produção de 0,7 g/frasco de matéria seca de plântulas de orquídea; ${ }^{(2)}$ e ${ }^{(3)}$ requerimento de nutrientes que indica as dose de nutrientes a adicionar em $40 \mathrm{~mL}$ por frasco ou por $\mathrm{L}$ de meio de cultura.

Quadro 2. Teores e demandas pelas plântulas de orquídea e requerimentos de nutrientes a serem adicionados no meio de cultura com o novo Fertilizante-Suprimento (FS2)

\begin{tabular}{|c|c|c|c|c|c|c|c|c|c|}
\hline Variável & Unidade & $\mathbf{N}$ & $\mathbf{P}$ & $\mathbf{S}$ & $\mathbf{K}$ & $\mathrm{Ca}$ & B & $\mathrm{Zn}$ & $\mathbf{F e}$ \\
\hline$t t \mathrm{Nu}_{\mathrm{i}}$ & $\mathrm{g} \mathrm{kg}^{-1}$ & 15,000 & 7,000 & 3,050 & 22,100 & 12,000 & 0,100 & 0,100 & 0,120 \\
\hline$d \mathrm{Nu}_{\mathrm{i}}^{(1)}$ & $\mathrm{mg} /$ frasco & 13,500 & 6,300 & 2,745 & 19,890 & 10,800 & 0,090 & 0,090 & 0,108 \\
\hline $\operatorname{tr} \mathrm{Nu}_{\mathrm{i}}-\mathrm{Pl}$ & & 0,850 & 0,800 & 0,550 & 0,750 & 0,900 & 0,800 & 0,900 & 0,300 \\
\hline$r \mathrm{Nu}_{\mathrm{i}}^{(2)}$ & $\mathrm{mg} /$ frasco & 15,882 & 7,875 & 4,991 & 26,520 & 12,000 & 0,113 & 0,100 & 0,360 \\
\hline$r \mathrm{Nu}_{\mathrm{i}}^{(3)}$ & $m g \mathrm{~L}^{-1}$ & 397,059 & 196,875 & 124,773 & 663,000 & 300,000 & 2,813 & 2,500 & 9,000 \\
\hline
\end{tabular}

(1) Demanda de nutrientes para produção de 0,9 g/frasco de matéria seca de plântulas de orquídea; (2) e (3) requerimento de nutrientes que indica as dose de nutrientes a adicionar em $40 \mathrm{~mL}$ por frasco ou por L de meio de cultura. 
$\mathrm{O} s \mathrm{Nu}_{\mathrm{i}}$ corresponde ao suprimento do substrato $\left(s \mathrm{Nu}_{\mathrm{i}}-\mathrm{Sb}\right)$ mais o suprimento do nutriente adicionado via fertilizante até a época da colheita $\left(s \mathrm{Nu}_{\mathrm{i}}-\mathrm{FS}\right): s \mathrm{Nu}_{\mathrm{i}}=s \mathrm{Nu}_{\mathrm{i}}-\mathrm{Sb}+s \mathrm{Nu}_{\mathrm{i}}-\mathrm{FS}$.

A demanda dos nutrientes (Quadro 3) e o suprimento total dos nutrientes (Quadro 4) permitiram calcular as taxas de recuperação dos nutrientes pelas plantas em cada época de colheita: $\operatorname{tr} \mathrm{Nu}_{\mathrm{i}}-\mathrm{Pl}=d \mathrm{Nu}_{\mathrm{i}} / s \mathrm{Nu}_{\mathrm{i}}$ (Quadro 5). As taxas de recuperação são elevadas porque no cálculo do suprimento total foram considerados os teores disponíveis de nutrientes nas análises químicas do substrato (escala de teores) e não a real disponibilidade dos nutrientes às plantas (escala de doses), demonstrado na terceira tentativa. $\mathrm{O} r \mathrm{Nu}_{\mathrm{i}}$ indica as doses dos $\mathrm{Nu}_{\mathrm{i}}$ para duas aplicações entre épocas de colheita do novo FS (FS2) (Quadro 6).

As doses de nutrientes no FS2 foram determinadas com as médias para duas aplicações entre épocas de colheita. Para N, as doses diminuíram de 16,00 a $7,15 \mathrm{mg} / \mathrm{vaso} /$ aplic, com média de $11,587 \mathrm{mg} / \mathrm{vaso} / \mathrm{aplic}$ e total em 14 aplicações de 162,211 mg/vaso; ou seja, $115,87 \mathrm{mg} / \mathrm{vaso} /$ aplic de FS2, na forma de $25 \mathrm{~mL} / \mathrm{vaso} / a$ plic. Entretanto, a proporção entre nutrientes manteve-se constante no FS2. Essa proporção, em \% (m/m), foi: 10,00 N; 4,832 P; 4,415 S; 12,948 K;
7,765 Ca; 3,410 Mg; 0,101 B; 0,005 Mo; 0,294 Zn; 0,980 Fe; 0,492 Mn; e 0,047 Cu. Para preparar a solução do FS2, foi necessário pesar 11,587 g do fertilizante multinutriente FS2, dissolver e diluir até 2,5 L. Dessa solução foram aplicados $25 \mathrm{~mL} /$ vaso/aplic.

$\mathrm{Na}$ segunda tentativa, não foram consideradas as $t r \mathrm{Nu}_{\mathrm{i}}$-Ex, pois utilizaram-se os teores disponíveis de nutrientes do substrato. Na terceira tentativa, já foi possível determinar a primeira aproximação dessas taxas.

\section{Terceira tentativa: Cultivo de violetas em substrato}

A terceira tentativa foi realizada para determinar a taxa de recuperação de macro e micronutrientes pelos extratores de formas disponíveis, a demanda e a taxa de recuperação de nutrientes por plantas de violeta africana (Saintpaulia ionantha Wendl) e a dose e os teores de nutrientes no fertilizante FS que suplementam seus requerimentos (Matta, 2012).

O substrato foi composto por uma parte de areia, uma de solo, uma de casca de arroz carbonizada e uma de fibra de coco. O substrato foi preparado por repetição. A média dos resultados das análises químicas apresenta-se no quadro 7. As mudas foram

Quadro 3. Demanda de N, P, S, K, Ca, B, Zn e Fe de plantas de violeta aos 21, 84 e 147 d

\begin{tabular}{|c|c|c|c|c|c|c|c|c|}
\hline Época ${ }^{(1)}$ & $\mathbf{N}^{(2)}$ & $\mathbf{P}$ & $\mathbf{S}$ & $\mathbf{K}$ & $\mathbf{C a}$ & B & $\mathrm{Zn}$ & $\mathbf{F e}$ \\
\hline$d$ & \multicolumn{8}{|c|}{ - mg/planta } \\
\hline 21 & 11,450 & 2,510 & 1,560 & 33,170 & 10,120 & 0,102 & 0,094 & 0,440 \\
\hline 84 & 64,060 & 18,380 & 7,335 & 124,670 & 45,765 & 0,335 & 0,414 & 1,936 \\
\hline 147 & 88,340 & 23,690 & 11,430 & 178,870 & 66,130 & 0,549 & 0,750 & 2,534 \\
\hline
\end{tabular}

(1) As épocas de colheita estudadas foram: 21, 42, 63, 84, 105, 126 e 147 d; e ${ }^{(2)}$ os nutrientes: N, P, S, K, Ca, Mg, B, Mo, Fe, Zn, Mn e $\mathrm{Cu}$.

Quadro 4. Suprimento total dos nutrientes via substrato e fertilizante adicionado até a época de colheita

\begin{tabular}{ccccccccc}
\hline Época $^{(1)}$ & $\mathbf{N}$ & $\mathbf{P}$ & $\mathbf{S}$ & $\mathbf{K}$ & $\mathbf{C a}$ & $\mathbf{B}$ & $\mathbf{Z n}$ & $\mathbf{F e}$ \\
\hline \multirow{2}{*}{$\mathrm{d}$} & & & \multicolumn{7}{c}{ mg/planta } \\
\cline { 2 - 7 } 21 & 27,990 & 15,950 & 13,900 & 40,324 & 24,190 & 0,303 & 0,923 \\
84 & 106,770 & 51,480 & 47,150 & 137,247 & 82,318 & 1,047 & 3,150 & 10,573 \\
147 & 155,170 & 75,040 & 68,500 & 210,030 & 125,950 & 1,628 & 4,557 & 15,152 \\
\hline
\end{tabular}

(1) As épocas de colheita estudadas foram: 21, 42, 63, 84, 105, 126 e $147 \mathrm{~d}$.

Quadro 5. Taxas de recuperação dos nutrientes pelas plantas na época de colheita

\begin{tabular}{|c|c|c|c|c|c|c|c|c|}
\hline Época & $\mathbf{N}$ & $\mathbf{P}$ & $\mathrm{S}$ & $\mathbf{K}$ & $\mathbf{C a}$ & $\mathbf{B}$ & $\mathrm{Zn}$ & $\mathbf{F e}$ \\
\hline $\mathrm{d}$ & \multicolumn{8}{|c|}{$(\mathrm{mg} /$ planta $) /(\mathrm{mg} / \mathrm{planta})$} \\
\hline 21 & 0,409 & 0,157 & 0,112 & 0,823 & 0,418 & 0,337 & 0,102 & 0,162 \\
\hline 84 & 0,600 & 0,357 & 0,156 & 0,908 & 0,556 & 0,320 & 0,131 & 0,183 \\
\hline 147 & 0,569 & 0,316 & 0,167 & 0,852 & 0,525 & 0,337 & 0,165 & 0,167 \\
\hline
\end{tabular}


transplantadas no mesmo dia em que chegaram ao Departamento de Solos/UFV.

O experimento foi conduzido em casa de vegetação. A unidade experimental foi composta por um prato com três vasos com $0,4 \mathrm{dm}^{3}$ de substrato, com uma planta por vaso.

O arranjo experimental utilizado foi um fatorial $2((6 \times 4)+1)$, duas variedades de violeta, Kalisa (Krs) e Fiesta (Fba), cultivadas com seis doses e quatro épocas de colheita mais o tempo zero correspondente à amostragem feita nas mudas no dia em que chegaram ao DPS/UFV. As doses estudadas foram 0 , 20, 40, 60, 120 e $200 \mathrm{mg} / \mathrm{dm}^{3} /$ aplic de FS1, aplicadas em solução. Para aplicar a dose a $20 \mathrm{mg} / \mathrm{dm}^{3} /$ aplic, foram realizadas duas diluições: a primeira, $6 \mathrm{~g} / 2,5 \mathrm{~L}$ de FS1 (FS1 diluído) e, a segunda, para aplicação, 10 $\mathrm{mL}$ de FS1, diluídos até $100 \mathrm{~mL}$ de solução para aplicação do tratamento. A cada 7 d, 100 mL de FS1 em solução, de acordo com as doses, foram aplicadas por unidade experimental. A aplicação das doses de FS1 implica em aplicação de doses variáveis de macro e micronutrientes, variando concomitantemente entre eles de acordo com as doses de FS1 (Quadro 8). As quatro épocas de colheita foram 35, 70, 105 e $140 \mathrm{~d}$, após o início da aplicação das doses de fertilizante, mais o tempo zero, correspondente à amostragem feita em mudas antes da instalação do experimento.

A fertilização foi feita nos pratos uma vez por semana; e a irrigação, de uma a duas vezes por semana, de acordo com a necessidade da planta de modo a não permitir umidade excessiva.

Em cada época de colheita, foram coletadas as partes aéreas das plantas, que foram separadas em meristemas e folhas; a partir da segunda colheita $(70 \mathrm{~d})$, começou-se a separar também as inflorescências. Os nutrientes estudados foram: N, $\mathrm{P}, \mathrm{S}, \mathrm{K}, \mathrm{Ca}, \mathrm{Mg}, \mathrm{B}, \mathrm{Mo}, \mathrm{Fe}, \mathrm{Zn}, \mathrm{Mn}$ e $\mathrm{Cu}$.

Em cada colheita foi retirada uma amostra do substrato de cada unidade experimental, para análise química.

A parte vegetal (folhas, meristema e inflorescências) foi seca em estufa de circulação forçada a $70^{\circ} \mathrm{C}$, pesada, moída e mineralizada por via seca. Em seguida, o S, $\mathrm{Ca}, \mathrm{Mg}, \mathrm{B}, \mathrm{Mo}, \mathrm{Zn}, \mathrm{Fe}, \mathrm{Mn}$ e $\mathrm{Cu}$ foram dosados por espectrofotômetria de emissão ótica em plasma induzido (ICP-OES), o $\mathrm{P}$ foi por colorimetria, o K, por fotometria de emissão em chama e o N, pelo método Kjeldahl.

A partir do teor total do nutriente i no órgão vegetal $\mathrm{j}\left(t t \mathrm{Nu}_{\mathrm{ij}}\right)$ e da produção de matéria seca de cada órgão vegetal $\left(m \mathrm{MS}_{\mathrm{j}}\right)$ da planta, foram obtidos o conteúdo do nutriente i do órgão vegetal $\mathrm{j}:\left(c \mathrm{Nu}_{\mathrm{ij}}=t t \mathrm{Nu}_{\mathrm{ij}} \times m \mathrm{MS}_{\mathrm{j}}\right)$ e, pelo somatório, o conteúdo total do nutriente na planta $\left(c \mathrm{Nu}_{\mathrm{i}}=c \mathrm{Nu}_{\mathrm{i}}-\right.$ Meristema $+c \mathrm{Nu}_{\mathrm{i}}$-Folhas $+c \mathrm{Nu}_{\mathrm{i}}$-Inflorescências).

A produção total de matéria seca por planta, assim como os conteúdos totais de nutrientes, para cada dose de FS1, em função das épocas de colheita, se ajustou bem ao modelo de crescimento logístico. Esse modelo supõe que a produção cresce até um determinado limite, quando tende a se estabilizar e posteriormente a decrescer. Ambas as variedades acumularam mais matéria seca nas doses 120 e $200 \mathrm{mg} / \mathrm{dm}^{3} /$ aplic de FS1. Na dose $120 \mathrm{mg} / \mathrm{dm}^{3} /$ aplic, para a variedade Kaliza (Krs), a função de crescimento foi: $\hat{y}=2,4612 /\left(1+26,6343 \mathrm{e}^{-0,0455 t}\right)$ e $\mathrm{R}^{2}=0,997$; e, para a variedade Fiesta (Fba): $\hat{y}=2,8962 /\left(1+24,6059 \mathrm{e}^{-0,0389 t}\right)$ e $R^{2}=0,995$. A diferença de acúmulo de massa de matéria seca não foi marcante entre as duas variedades dentro da mesma dose (Matta, 2012).

Quadro 6. Requerimento dos nutrientes pelas plantas entre épocas de colheita

\begin{tabular}{|c|c|c|c|c|c|c|c|c|}
\hline Época $^{(1)}$ & $\mathbf{N}^{(2)}$ & $\mathbf{P}$ & $\mathrm{S}$ & $\mathbf{K}$ & $\mathbf{C a}$ & B & $\mathrm{Zn}_{\mathbf{n}}$ & $\mathbf{F e}$ \\
\hline \multicolumn{9}{|c|}{ - mg/planta } \\
\hline $0-42^{(1)}$ & 28,865 & 14,615 & 13,400 & 40,324 & 24,190 & 0,303 & 0,896 & 2,518 \\
\hline $42-84^{(2)}$ & 24,520 & 11,125 & 10,175 & 29,213 & 17,518 & 0,227 & 0,679 & 2,769 \\
\hline $84-147^{(3)}$ & 16,133 & 7,853 & 7,117 & 22,053 & 13,218 & 0,179 & 0,469 & 1,526 \\
\hline Média & 23,173 & 11,198 & 10,231 & 30,004 & 17,993 & 0,233 & 0,681 & 2,271 \\
\hline
\end{tabular}

(1) Requerimento médio para as duas primeiras épocas de colheita: 0 a 21 e 21 a 42 d (quatro aplicações); ${ }^{(2)}$ requerimento médio para 42 a 63 e 63 a 84 d; e ${ }^{(3)}$ requerimento médio para três épocas, 84 a 105; 105 a 126; e 126 a $147 \mathrm{~d}$ (seis aplicações).

Quadro 7. Caracterização química do substrato, médias de quatro repetições

\begin{tabular}{|c|c|c|c|c|c|c|c|c|c|c|c|}
\hline $\mathrm{pH}\left(\mathrm{H}_{2} \mathrm{O}\right)$ & $\mathbf{P}$ & $\mathbf{S}$ & $\mathbf{K}$ & $\mathbf{C a}$ & Mg & B & $\mathrm{Zn}$ & $\mathbf{F e}$ & Mn & $\mathrm{Cu}$ & MO \\
\hline & & & & & $\mathrm{m}$ & $m^{-3}-$ & . & 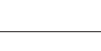 & & 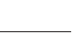 & $\mathrm{g} \mathrm{kg}^{-1}$ \\
\hline 6,01 & 33,08 & 32,13 & 628,00 & 88,00 & 33,41 & 0,24 & 5,80 & 128,35 & 36,80 & 1,28 & 61,25 \\
\hline
\end{tabular}

Extratores utilizados: P, K, Fe, Zn, Mn e Cu - Mehlich 1; Ca e Mg - KCl 1 mol/L; B - água quente; S - Fosfato monocálcico em ácido acético; e MO - Matéria orgânica - Walkley-Black. 
Em relação aos conteúdos de macro e micronutrientes, percebeu-se que a variedade Fba foi aquela que acumulou a maior quantidade de nutrientes, exceto para o teor de Fe, que foi superado pela variedade Krs (Matta, 2012).

As quatro épocas de colheita apresentaram teores nas plantas que seguem uma mesma tendência. Para calcular as taxas de recuperação dos extratores e das plantas e determinar as doses e os teores de nutrientes do novo FS (FS2), foram consideradas aquelas encontradas na terceira colheita, por essa representar a época em que a violeta está por atingir o platô da curva de crescimento (variedade Krs 2,01 e Fba 2,05 $\mathrm{g} /$ planta de matéria seca) e por encontrar-se no ponto de interesse comercial.

Para o cálculo das taxas de recuperação dos extratores, foram ajustadas equações das quantidades de macro e micronutrientes extraídas do substrato (y, em mg/vaso), após 15 aplicações das doses de nutrientes no FS1 (x, em mg/vaso). Por exemplo, para $\mathrm{P}$ a equação foi: $\hat{y}=7,15+0,0879^{* *} \mathrm{x}+0,0098^{*} \mathrm{x}^{2}$, $R^{2}=0,992$. Essa equação indica que a taxa específica de recuperação de $\mathrm{P}$ por Mehlich-1 do $\mathrm{P}$ adicionado ao substrato ( $t r \mathrm{P}-\mathrm{M} 1)$ foi variável com a dose: para a dose 0 foi 0,088; para a dose 19,005 (15×1,267, Quadro 8), 0,464 ; e, para a dose $31,68,0,715$. Em razão dessa ampla variação das $t r \mathrm{Nu}_{\mathrm{i}}$-Ex se preferiu estimar as taxas para o intervalo entre a dose 0 e a dose para produção máxima.

A função de produção de matéria seca das plantas de violeta em resposta às doses de FS1 na variedade Krs foi: $\hat{y}=1,25+0,01195^{* *} \mathrm{x}-0,0000397^{*} \mathrm{x}^{2}, \mathrm{R}^{2}$ $=0,991 ;$ e na variedade Fba: $\mathrm{w}=1,22+0,0131^{* *} \mathrm{x}$ $-0,0000469^{* *} x^{2}, R^{2}=0,993$. As doses para produção máxima foram 150,5 e $139,7 \mathrm{mg} / \mathrm{dm}^{3} /$ aplic; e as produções máximas foram 2,15 e 2,14 g/planta, respectivamente, para as variedades Krs e Fba.

Para continuar com o cálculo das $t r \mathrm{Nu}_{\mathrm{i}}$-Ex, consideraram-se as quantidades de nutrientes adicionadas com a dose para produção máxima das plantas da variedade Fba, $140 \mathrm{mg} / \mathrm{dm}^{3} /$ aplic do FS1. Das equações para estimar a quantidade de macro e micronutrientes extraídos do substrato, na terceira época de colheita, em função das doses de nutrientes adicionados (Quadro 8), em 15 aplicações, foram estimadas as quantidades recuperadas do substrato para as doses de 0 e $140 \mathrm{mg} / \mathrm{dm}^{3} /$ aplic do FS1 (Quadro 9).

Como o requerimento do nutriente pela planta é obtido pela divisão da demanda nutricional pela taxa de recuperação do nutriente pela planta $\left(r \mathrm{Nu}_{\mathrm{i}}=d \mathrm{Nu}_{\mathrm{i}} / t r \mathrm{Nu}_{\mathrm{i}} \mathrm{Pl}\right)$, é imprescindível calcular essas taxas. As taxas de recuperação dos nutrientes pela planta $\left(t r \mathrm{Nu}_{\mathrm{i}} \mathrm{Pl}=d \mathrm{Nu}_{\mathrm{i}} / s \mathrm{Nu}_{\mathrm{i}}\right)$ foram calculadas de três formas diferentes ao se utilizarem três critérios para se obter $s \mathrm{Nu}_{\mathrm{i}}$, a quantidade do $\mathrm{Nu}_{\mathrm{i}}$ disponibilizada no substrato ( $\left.q d \mathrm{Nu}_{\mathrm{i}}-\mathrm{Sb}\right)$. As $d \mathrm{Nu}_{\mathrm{i}}$ foram calculadas com base nas equações para estimar os conteúdos totais em função das doses de nutrientes aplicadas no substrato: $d \mathrm{Nu}_{\mathrm{i}}=\left(d \mathrm{Nu}_{\mathrm{ik}}-d \mathrm{Nu}_{\mathrm{i} 0}\right)$,

em que: $d \mathrm{Nu}_{\mathrm{ik}}=$ demanda do nutriente i pelas plantas fertilizadas com a dose $\mathrm{k}_{\max }$, para a variedade Fba; a terceira colheita foi considerada a dose para produção máxima de $140 \mathrm{mg} / \mathrm{vaso}$ de $\mathrm{FS} 1$; e $d \mathrm{Nu}_{\mathrm{i} 0}=$ demanda do nutriente i pelas plantas na dose 0 , em $\mathrm{mg} / \mathrm{vaso}$ (Quadro 10), e o suprimento do nutriente i disponibilizado pelo substrato, considerando: a) quantidade do nutriente i disponibilizada pela aplicação da dose $\mathrm{k}_{\max }\left(q d \mathrm{Nu}_{\mathrm{i}}-\mathrm{D}_{\mathrm{k}}\right)$; b) quantidade disponibilizada do nutriente $\mathrm{i}$ pela aplicação da dose $\mathrm{k}_{\max }$ mais o extraído do substrato pelas análises químicas, expressa na escala de teores $\left(q d \mathrm{Nu}_{\mathrm{i}}-\mathrm{D}_{\mathrm{k}}+\mathrm{Sb}\right)$; e c) quantidade disponibilizada do nutriente i pela aplicação da dose $\mathrm{k}_{\max }$ mais o extraído do substrato pelas análises químicas, mas corrigida pelas $t r \mathrm{Nu}_{\mathrm{i}}-\mathrm{Ex}$, para ser expressa na escala de doses $\left(q d \mathrm{Nu}_{\mathrm{i}}-\mathrm{D}_{\mathrm{k}}+\mathrm{Sb}_{\mathrm{c}}\right)$. Assim, foram calculadas três formas das taxas de recuperação dos nutrientes pela planta (Quadro 10):

Quadro 8. Quantidades de nutrientes aplicadas por vaso e por aplicação em cada dose de FS1

\begin{tabular}{|c|c|c|c|c|c|c|c|c|c|}
\hline \multirow{2}{*}{$\begin{array}{l}\text { Dose } \\
\text { FS1 }^{(3)} \\
\end{array}$} & \multirow{2}{*}{$\begin{array}{l}\text { Aliq }^{(1)} \\
\text { FS1d }^{(4)}\end{array}$} & \multicolumn{8}{|c|}{ Nutriente $^{(2)}$} \\
\hline & & $\mathbf{N}$ & $\mathbf{P}$ & $\mathbf{S}$ & $\mathbf{K}$ & $\mathbf{C a}$ & B & $\mathrm{Zn}$ & $\mathrm{Fe}$ \\
\hline $\mathrm{mg} / \mathrm{dm}^{3} /$ aplic & $\mathrm{mL}$ & & & $\mathrm{mg} / \mathrm{vas}$ & & & & $\mu \mathrm{g} / \mathrm{vaso}$ & \\
\hline 0 & 0 & 0,000 & 0,000 & 0,000 & 0,000 & 0,000 & 0,00 & 0,00 & 0,00 \\
\hline 20 & 10 & 0,800 & 0,211 & 0,202 & 0,681 & 0,168 & 4,88 & 9,60 & 28,80 \\
\hline 40 & 20 & 1,600 & 0,422 & 0,403 & 1,362 & 0,336 & 9,76 & 19,20 & 57,60 \\
\hline 80 & 40 & 3,200 & 0,845 & 0,816 & 2,723 & 0,672 & 19,52 & 38,40 & 115,20 \\
\hline 120 & 60 & 4,800 & 1,267 & 1,210 & 4,085 & 1,008 & 29,28 & 57,60 & 172,80 \\
\hline 200 & 100 & 8,000 & 2,112 & 2,016 & 6,808 & 1,680 & 48,80 & 96,00 & 288,00 \\
\hline
\end{tabular}

(1) Cada aplicação foi de $100 \mathrm{~mL}$ por unidade experimental (três vasos com $0,4 \mathrm{dm}^{3}$ de substrato $=1,2 \mathrm{dm}^{3}$ ); para preparar os 100 $\mathrm{mL}$ foram usadas essas alíquotas em função das doses. ${ }^{(2)} \mathrm{A}$ quantidade de cada nutriente refere-se a uma aplicação por vaso; os números de aplicações foram 5, 10, 15 e 20 para as épocas de colheita aos 35, 70, 105 e $140 \mathrm{~d}$, respectivamente. (3) O adubo FS1 continha, em \% (m/m), $10 \mathrm{~N} ; 2,64 \mathrm{P} ; 2,52 \mathrm{~S} ; 8,51 \mathrm{~K} ; 2,10 \mathrm{Ca} ; 1,21 \mathrm{Mg} ; 0,061 \mathrm{~B} ; 0,002 \mathrm{Mo}$; 0,12 Zn; 0,36 Fe; 0,14 Mn; e 0,015 Cu. (4) $\mathrm{O}$ adubo FS1 diluído continha, em mg/L, N 240,0; P 63,4; S 60,5; K 204,2; Ca 50,4; Mg 29,0; B 1,5; Mo 0,048; Zn 2,9; Fe 8,6; $\mathrm{Mn} 3,4$; e $\mathrm{Cu} 0,4$. 
a) $t r \mathrm{Nu}_{\mathrm{i}}-\mathrm{Pl}, \mathrm{D}_{\mathrm{k}}=d \mathrm{Nu}_{\mathrm{i}} / q d \mathrm{Nu}_{\mathrm{i}}-\mathrm{D}_{\mathrm{k}}$;

b) $t r \mathrm{Nu}_{\mathrm{i}}-\mathrm{Pl}, \mathrm{D}_{\mathrm{k}}+\mathrm{Sb}=d \mathrm{Nu}_{\mathrm{i}} / q d \mathrm{Nu}_{\mathrm{i}}-\mathrm{D}_{\mathrm{k}}+\mathrm{Sb} ; \mathrm{e}$

c) $t r \mathrm{Nu}_{\mathrm{i}}-\mathrm{Pl}, \mathrm{D}_{\mathrm{k}}+\mathrm{Sb}_{\mathrm{c}}=d \mathrm{Nu}_{\mathrm{i}} / q d \mathrm{Nu}_{\mathrm{i}}-\mathrm{D}_{\mathrm{k}}+\mathrm{Sb}_{\mathrm{c}}$.

A contribuição do substrato na disponibilização de nutrientes às plantas de violeta foi maior do que era adicionado com o FS1 para $\mathrm{P}, \mathrm{S}$ e $\mathrm{Cu}$ e muito maior para $\mathrm{K}, \mathrm{Ca}, \mathrm{Mg}, \mathrm{Zn}, \mathrm{Fe}$ e $\mathrm{Mn}$. Por isso, as $t r \mathrm{Nu}_{\mathrm{i}}-\mathrm{Pl}$, considerando unicamente as quantidades de nutrientes adicionadas com a dose $140 \mathrm{mg} / \mathrm{dm}^{3} / \mathrm{aplic}$ do FS1 $\left(q d \mathrm{Nu}_{\mathrm{i}}-\mathrm{D}_{\mathrm{k}}\right)$, foram muito elevadas, em comparação às taxas, analisando a contribuição da dose $140 \mathrm{mg} / \mathrm{dm}^{3} /$ aplic do FS e dos nutrientes do substrato, especialmente quando corrigidos pelas $\operatorname{tr} \mathrm{Nu}_{\mathrm{i}}-\mathrm{Ex}\left(q d \mathrm{Nu}_{\mathrm{i}}-\mathrm{D}_{\mathrm{k}}+\mathrm{Sb}_{\mathrm{c}}\right)$. As $\operatorname{tr} \mathrm{Nu}_{\mathrm{i}}-\mathrm{Pl}, \mathrm{D}_{\mathrm{k}}$ foram mais de 20 vezes maiores para $\mathrm{Fe}$ e $\mathrm{Mn}$, de 6 a 8 vezes para $\mathrm{Zn}$ e $\mathrm{Cu}$ e de 2 a 5 vezes para $\mathrm{K}$, Ca e $\mathrm{Mg}$ do que as $t r \mathrm{Nu}_{\mathrm{i}}-\mathrm{Pl}, \mathrm{D}_{\mathrm{k}}+\mathrm{Sb}_{c}$; inclusive para $\mathrm{Ca}$ e $\mathrm{Mg}$ as $t r \mathrm{Nu}_{\mathrm{i}}-\mathrm{Pl}, \mathrm{D}_{\mathrm{k}}$ foram maiores a $100 \%$.

As $t r \mathrm{Nu}_{\mathrm{i}}-\mathrm{Pl}, \mathrm{D}_{\mathrm{k}}+\mathrm{Sb}$ foram mais de 2 vezes maiores para $\mathrm{Zn}$ do que as $\operatorname{tr} \mathrm{Nu}_{\mathrm{i}}-\mathrm{Pl}, \mathrm{D}_{\mathrm{k}}+\mathrm{Sb}_{\mathrm{c}}$; de 1,5 a 2,0 vezes maiores para $\mathrm{P}, \mathrm{Mg}, \mathrm{Fe}$ e $\mathrm{Mn}$; e de 1,0 a 1,5 vez maior para $\mathrm{S}, \mathrm{K}, \mathrm{Ca}, \mathrm{B}$ e $\mathrm{Cu}$.
A utilização de $q d \mathrm{Nu}_{\mathrm{i}} \mathrm{D}_{\mathrm{k}}+\mathrm{Sb}$ para cálculo das $t r \mathrm{Nu}_{\mathrm{i}}-\mathrm{Pl}, \mathrm{D}_{\mathrm{k}}+\mathrm{Sb}$ seria aceitável caso se desconhecessem as $t r \mathrm{Nu}_{\mathrm{i}}-\mathrm{Ex}$, pois os bons extratores de formas disponíveis extraem o nutriente do fator intensidade e parte do fator quantidade (I + pQ); para alguns extratores e nutrientes, p é inversamente proporcional à capacidade tampão (CT).

A alta taxa de recuperação de $\mathrm{N}$ na terceira colheita pode estar relacionada a uma possível imobilização de N pela MOS nas primeiras épocas de aplicação/colheita, com mineralização da segunda a terceira época de colheita, disponibilizando $\mathrm{N}$ para as plantas. A baixa taxa de recuperação de $\mathrm{S}$ pode ser por causa de o processo de mineralização na análise foliar (calcinação) não ter sido eficiente para a determinação desse elemento (Matta, 2012). As baixas taxas de recuperação de nutrientes pela planta, principalmente para micronutrientes cátions, podem estar relacionadas com uma baixa eficiência do sistema radicular que é extremamente pequeno em plantas de violeta $(\approx 1 \%$ da $m \mathrm{MS}$ total) e, pela forma de aplicação das doses de FS1, adicionadas em solução nos pratos com as UE, pelo que para atingir as raízes

Quadro 9. Quantidades disponíveis extraídas do substrato, quantidades adicionadas com o fertilizante FS1 e taxas de recuperação por extratores de macro e micronutrientes, na terceira época de colheita

\begin{tabular}{llcrrrrrrr}
\hline Variável & Unid. & $\mathbf{N}$ & $\mathbf{P}$ & $\mathbf{S}$ & $\mathbf{K}$ & $\mathbf{C a}$ & $\mathbf{B}$ & $\mathbf{Z n}$ & $\mathbf{F e}$ \\
\hline$q r \mathrm{Nu}_{\mathrm{i} 0}-\mathrm{Sb}^{(1)}$ & $\mathrm{mg} /$ vaso & - & 7,150 & 32,006 & 166,250 & 117,630 & 0,189 & 1,594 & 32,424 \\
$q r \mathrm{Nu}_{\mathrm{i} 140}-\mathrm{Sb}^{(1)}$ & $\mathrm{mg} /$ vaso & - & 13,854 & 41,925 & 235,047 & 128,737 & 0,423 & 1,991 & 34,009 \\
$q a \mathrm{Nu}_{\mathrm{i} 140}-\mathrm{FS}^{(2)}$ & $\mathrm{mg} /$ vaso & 84,000 & 22,176 & 21,168 & 71,484 & 17,640 & 0,512 & 1,008 & 3,024 \\
$t r \mathrm{Nu}_{\mathrm{i}}-\mathrm{Ex}^{(3)}$ & & $0,800^{(4)}$ & 0,302 & 0,469 & 0,962 & 0,630 & 0,456 & 0,394 & 0,524 \\
\hline
\end{tabular}

(1) $q r$ = quantidade recuperada do $\mathrm{Nu}_{\mathrm{i}}$ pelos extratores do substrato na dose 0 e na dose $140 ;{ }^{(2)} q a=$ quantidades adicionada do $\mathrm{Nu}_{\mathrm{i}}$ na dose 140 do FS1; ${ }^{(3)}$ trNui-Ex = taxas de recuperação dos nutrientes pelos extratores. ${ }^{(4)}$ Como não foi analisado N, considerouse esta taxa.

Quadro 10. Demanda dos nutrientes pelas plantas fertilizadas com as doses 0 e $140 \mathrm{mg} / \mathrm{dm} /$ aplic do FS1, quantidades disponibilizadas de nutrientes pela adição da dose $140 \mathrm{mg} / \mathrm{dm}^{3} / \mathrm{aplic}$ do FS1 e a disponibilizada pelo substrato e as taxas de recuperação para a variedade Fiesta branca e azul, na terceira colheita

\begin{tabular}{|c|c|c|c|c|c|c|c|c|c|}
\hline Variável & Unid. & $\mathbf{N}$ & $\mathbf{P}$ & $\mathbf{S}$ & $\mathbf{K}$ & $\mathrm{Ca}$ & B & $\mathrm{Zn}$ & $\mathbf{F e}$ \\
\hline$d \mathrm{Nu}_{\mathrm{i} 0}$ & $\mathrm{mg} /$ planta & 20,020 & 7,223 & 2,021 & 63,010 & 16,370 & 0,042 & 0,081 & 0,018 \\
\hline$d \mathrm{Nu}_{\mathrm{i} 140}$ & $\mathrm{mg} /$ planta & 101,290 & 20,466 & 4,493 & 101,604 & 39,874 & 0,091 & 0,263 & 0,540 \\
\hline$d \mathrm{Nu}_{\mathrm{i}}$ & $\mathrm{mg} /$ planta & 81,270 & 13,243 & 2,472 & 38,594 & 23,504 & 0,049 & 0,182 & 0,523 \\
\hline$q d \mathrm{Nu}_{\mathrm{i}}-\mathrm{D}_{\mathrm{k}}$ & mg/vaso & 84,000 & 22,176 & 21,170 & 71,484 & 17,640 & 0,512 & 1,008 & 3,024 \\
\hline $\operatorname{tr} \mathrm{Nu}_{\mathrm{i}}-\mathrm{Pl}, \mathrm{D}_{\mathrm{k}}$ & & 0,968 & 0,597 & 0,117 & 0,540 & 1,332 & 0,095 & 0,180 & 0,173 \\
\hline$q d \mathrm{Nu}_{\mathrm{i}}-\mathrm{Sb}$ & $\mathrm{mg} / \mathrm{vaso}$ & 20,020 & 13,232 & 12,852 & 251,200 & 35,200 & 0,096 & 2,320 & 51,340 \\
\hline$q d \mathrm{Nu}_{\mathrm{i}}-\mathrm{D}_{\mathrm{k}}+\mathrm{Sb}$ & $\mathrm{mg} /$ vaso & 104,020 & 35,408 & 34,020 & 322,684 & 52,840 & 0,608 & 3,328 & 54,364 \\
\hline $\operatorname{tr}$ Nui-Pl, $\mathrm{D}_{\mathrm{k}}+\mathrm{Sb}$ & & 0,781 & 0,374 & 0,073 & 0,120 & 0,445 & 0,080 & 0,055 & 0,010 \\
\hline$q d \mathrm{Nu}_{\mathrm{i}}-\mathrm{Sb}_{\mathrm{c}}$ & $\mathrm{mg} /$ vaso & 25,025 & 43,524 & 27,480 & 260,710 & 55,903 & 0,211 & 5,883 & 82,253 \\
\hline$q d \mathrm{Nu}_{\mathrm{i}}-\mathrm{D}_{\mathrm{k}}+\mathrm{Sb}_{\mathrm{c}}$ & $\mathrm{mg} / \mathrm{vaso}$ & 109,025 & 65,700 & 48,649 & 332,189 & 73,543 & 0,723 & 6,891 & 85,277 \\
\hline $\operatorname{tr} \mathrm{Nu}_{\mathrm{i}}-\mathrm{Pl}, \mathrm{D}_{\mathrm{k}}+\mathrm{Sb}_{\mathrm{c}}$ & & 0,745 & 0,202 & 0,051 & 0,116 & 0,320 & 0,068 & 0,026 & 0,006 \\
\hline
\end{tabular}

(1) $d, q d, t r=$ demanda, quantidade disponibilizada e taxa de recuperação: $\mathrm{Nu}_{\mathrm{i}}=$ nutriente $\mathrm{i} ; \mathrm{D}_{\mathrm{k}}=$ quantidade de nutriente adicionada com a dose de $140 \mathrm{mg} / \mathrm{dm}^{3} /$ aplic do $\mathrm{FS} 1 ; \mathrm{Sb}=$ disponibilizada pelo substrato, na escala de teores; $\mathrm{Sb}_{\mathrm{c}}=\mathrm{disponibilizada}$ pelo substrato na escala de doses. 
os nutrientes precisam ascender por capilaridade. A aplicação de FS em solução deve ser no futuro na superfície do substrato.

Para produção de $2,14 \mathrm{~g} / \mathrm{planta}$ da variedade Fba, o requerimento de nutrientes depende da demanda de nutrientes corrigida pela taxa de recuperação pelas plantas, o que permitiu calcular a proporção de nutrientes no novo fertilizante FS (FS2) (Quadro 11).

Para produção de $2,14 \mathrm{~g} /$ planta da variedade Fba, em 15 semanas de cultivo, após transplantio das mudas, foi necessário adicionar 109,025 mg/vaso de $\mathrm{N}$, em 15 aplicações semanais, ou seja $145,367 \mathrm{mg} /$ vaso/aplic de FS2 em solução. O FS2 deve conter, em \% (m/m): 5,000 N; 3,013 P; 2,231 S; 15,235 K; 3,373 Ca; 1,683 Mg; 0,033 B; 0,001 Mo; 0,316 Zn; 3,911 Fe; 1,143 Mn e 0,043 Cu (Quadro 11). Para preparar a solução do FS2, foi necessário pesar $14,54 \mathrm{~g}$, dissolver e diluir até 2,5 L. Dessa solução, foram aplicados $25 \mathrm{~mL} / \mathrm{vaso} /$ aplic. Esse fertilizante ficou extremamente rico em micronutrientes cátions, em razão das muito pequenas taxas de recuperação pelas plantas para esses nutrientes.

\section{Técnica Experimental Projetada}

Essa técnica tem como objetivos determinar a curva de resposta a doses do fertilizante-suprimento definido preliminarmente (FS1), as taxas $t r \mathrm{Nu}_{\mathrm{i}} \mathrm{-Pl} \mathrm{e}$ $t r \mathrm{Nu}_{\mathrm{i}}$-Ex e a dose e os teores de nutrientes do fertilizante a ser recomendado (FS2).

Essa técnica contempla as seguintes etapas:

a) Determinação da dose e composição do FS1 a ser testado;

b) Condução do experimento;

c) Determinação da dose de FS1 para produção máxima;

d) Determinação das taxas de recuperação dos nutrientes pelos extratores de formas disponíveis $\left(t r \mathrm{Nu}_{\mathrm{i}}\right.$-Ex);

e) Determinação da demanda de nutrientes para produção máxima das plantas $\left(d \mathrm{Nu}_{\mathrm{i}}\right)$;

f) Determinação das taxas de recuperação dos nutrientes pelas plantas $\left(t r \mathrm{Nu}_{\mathrm{i}}-\mathrm{Pl}\right)$; g) Determinação do requerimento de nutrientes pelas plantas $\left(r \mathrm{Nu}_{\mathrm{i}}\right)$;

h) Determinação do suprimento (quantidade disponibilizada) de nutrientes pelo substrato $\left(q d \mathrm{Nu}_{\mathrm{i}^{-}}\right.$ $\mathrm{Sb}_{\mathrm{c}}$ ); e

i) Determinação das quantidades de nutrientes a serem suplementadas $\left(q s \mathrm{Nu}_{\mathrm{i}}-\mathrm{FS}\right)$ e os teores de nutrientes no FS2.

Para a):

Adquirir vasos com plantas ornamentais de qualidade comercial superior.

Formar várias amostras.

Dividir as plantas em seus compartimentos (órgãos vegetais).

Determinar a massa de matéria seca $\left(m \mathrm{MS}_{\mathrm{j}}\right)$ e o teor total dos nutrientes nos compartimentos das plantas $\left(t t \mathrm{Nu}_{\mathrm{ij}}\right)$.

Calcular os conteúdos dos nutrientes $\left(c \mathrm{Nu}_{\mathrm{ij}}\right)$ por compartimento e pela sua soma a demanda do nutriente $\left(d \mathrm{Nu}_{\mathrm{i}}\right)$ da planta.

Definir as taxas de recuperação dos nutrientes pelas plantas $\left(t r \mathrm{Nu}_{\mathrm{i}}-\mathrm{Pl}\right)$.

Calcular os requerimentos dos nutrientes $\left(r \mathrm{Nu}_{\mathrm{i}}\right)$.

Determinar os teores de formas disponíveis dos nutrientes do substrato $\left(t d \mathrm{Nu}_{\mathrm{i}}-\mathrm{Sb}\right)$.

Definir as taxas de recuperação dos nutrientes pelos extratores $\left(t r \mathrm{Nu}_{\mathrm{i}}\right.$-Ex).

Calcular o suprimento (quantidade disponibilizada) de nutrientes pelo substrato $\left(q d \mathrm{Nu}_{\mathrm{i}}-\mathrm{Sb}\right)$.

Calcular as quantidades a serem adicionadas de nutrientes do FS1, para suplementar os requerimentos das plantas $\left(q s \mathrm{Nu}_{\mathrm{i}}\right.$-FS1).

Determinar a dose e proporção de nutrientes do Fertilizante-Suprimento (FS1).

Parab), serão utilizados dois conjuntos de tratamentos:

a) Para determinar a curva de resposta a oito doses de FS1 e as $t r \mathrm{Nu}_{\mathrm{i}}$-Ex utilizam-se os seguintes níveis de FS1: 0,$00 ; 0,10 ; 0,25 ; 0,45 ; 0,70 ; 1,00 ; 1,35 ;$ e 1,75 . $\mathrm{O}$ nível 1,00 corresponde à dose de referência do FS1 completo (FS1C).

Quadro 11. Demanda e requerimento de nutrientes pelas plantas e teores de nutrientes no fertilizante FS2 para suplementar esses requerimentos

\begin{tabular}{lccccccccc}
\hline Variável & Unid. & $\mathbf{N}$ & $\mathbf{P}$ & $\mathbf{S}$ & $\mathbf{K}$ & $\mathbf{C a}$ & $\mathbf{B}$ & $\mathbf{Z n}$ & $\mathbf{F e}$ \\
\hline$d \mathrm{Nu}_{\mathrm{i}}-\mathrm{D}_{0}$ & $\mathrm{mg} /$ planta & 20,02 & 7,223 & 2,021 & 63,01 & 16,37 & 0,042 & 0,081 & 0,018 \\
$d \mathrm{Nu}_{\mathrm{i}}-\mathrm{D}_{140}$ & $\mathrm{~m}$ /planta & 101,29 & 20,466 & 4,493 & 101,604 & 39,874 & 0,091 & 0,263 & 0,540 \\
$d \mathrm{Nu}_{\mathrm{i}}-\mathrm{D}_{140-0}$ & $\mathrm{~m} /$ planta & 81,27 & 13,243 & 2,472 & 38,594 & 23,504 & 0,049 & 0,182 & 0,523 \\
$t r N u \mathrm{i}-\mathrm{Pl}, \mathrm{D}_{\mathrm{k}}+\mathrm{Sb}$ & & 0,745 & 0,202 & 0,051 & 0,116 & 0,32 & 0,068 & 0,026 & 0,006 \\
$q s \mathrm{Nu}_{\mathrm{i}}-\mathrm{FS} 2$ & $\mathrm{mg} / \mathrm{vaso}$ & 109,025 & 65,7 & 48,649 & 332,189 & 73,543 & 0,723 & 6,891 & 85,277 \\
$t \mathrm{Nu}_{\mathrm{i}}-\mathrm{FS} 2$ & $\%(\mathrm{~m} / \mathrm{m})$ & 5,000 & 3,013 & 2,231 & 15,235 & 3,373 & 0,033 & 0,316 & 3,911 \\
\hline
\end{tabular}

(1) $d, t r, q s, t=$ demanda, taxa de recuperação, quantidade a ser suplementada e teor; $\mathrm{Nu}_{\mathrm{i}}=$ nutriente i; e FS2 fertilizante para suplementar os requerimentos das plantas. 
b) Para determinar as taxas $t r \mathrm{Nu}_{\mathrm{i}} \mathrm{-Pl}$ utilizam-se oito tratamentos: FS1C; FS1C - N; FS1C - P - S; FS1C - K; FS1C - Ca - Mg; FS1C - B - Mo; FS1C - Fe - Mn; e FS1C - Zn - Cu.

Esses dois conjuntos de tratamentos formam a matriz experimental Baconiana.

A unidade experimental é de acordo com a cultura.

Delineamento experimental: blocos casualizados com quatro repetições.

$\mathrm{Na}$ época da colheita do experimento devem ser tomados os seguintes dados: $t d \mathrm{Nu}_{\mathrm{i}}-\mathrm{Sb}, m \mathrm{MS}_{\mathrm{j}}$ e $t t \mathrm{Nu}_{\mathrm{ij}}$.

Para c):

Estimar a equação de produção de MS por planta em função das doses de FS1. Pela primeira derivada igualada a 0 , determinar a dose para produção máxima $\left(\mathrm{k}_{\max }\right)$ e a produção máxima $\left(\hat{\mathrm{y}}_{\max }\right)$.

Para d):

Calcular as equações das quantidades de nutrientes recuperadas, do substrato, pelos extratores de formas disponíveis $\left(q d \mathrm{Nu}_{\mathrm{ik}}-\mathrm{Sb}\right)$, em função da quantidade adicionada do nutriente i na dose $\mathrm{k}$ do FS1 $\left(q a \mathrm{Nu}_{\mathrm{ik}}\right)$.

Estimar as quantidades de nutrientes recuperadas do substrato para as doses $0\left(q d \mathrm{Nu}_{\mathrm{i}} 0\right)$ e $\mathrm{k}_{\max }$ $\left(q d \mathrm{Nu}_{\mathrm{i}} \mathrm{k}_{\max }\right)$.

As $\operatorname{tr} \mathrm{Nu}_{\mathrm{i}}-\mathrm{Ex}$ devem ser estimadas de duas formas:

a) $t r \mathrm{Nu}_{\mathrm{i}}$-Ex $=$ as declividades das equações de regressão das quantidades de nutrientes recuperadas, do substrato, pelos extratores de formas disponíveis $\left(q d \mathrm{Nu}_{\mathrm{ik}}-\mathrm{Sb}\right)$ em função da quantidade adicionada do nutriente i na dose $\mathrm{k}$ do FS1 considerada $\left(q a \mathrm{Nu}_{\mathrm{i}} \mathrm{k}\right)$.

b) $t r \mathrm{Nu}_{\mathrm{i}}-\mathrm{Ex}=\left(q d \mathrm{Nu}_{\mathrm{i}} \mathrm{k}_{\max }-q d \mathrm{Nu}_{\mathrm{i}} 0\right) / q a \mathrm{Nu}_{\mathrm{i}} \mathrm{k}_{\max }$.

Para e):

Determinar a demanda de nutrientes para produção máxima das plantas. Calcular o conteúdo do nutriente i do compartimento j com base na $m \mathrm{MS}_{\mathrm{j}}$ e nos $t t \mathrm{Nu}_{\mathrm{ij}}: c \mathrm{Nu}_{\mathrm{ij}}=m \mathrm{MS}_{\mathrm{j}} \times t t \mathrm{Nu}_{\mathrm{ij}}$.

A demanda do nutriente i é o conteúdo total do nutriente i na planta: $c \mathrm{Nu}_{\mathrm{i}}=d \mathrm{Nu}_{\mathrm{i}}=\Sigma_{1}^{\mathrm{j}} c \mathrm{Nu}_{\mathrm{ij}}$.

Para f):

Calcular as equações da demanda do nutriente i da planta na dose $\mathrm{k}\left(d \mathrm{Nu}_{\mathrm{i}} \mathrm{k}\right)$ em função da quantidade adicionada do nutriente i na dose $\mathrm{k}$ do FS1 $\left(q a \mathrm{Nu}_{\mathrm{i}} \mathrm{k}\right)$.

Estimar as demandas do nutriente i para as doses $0\left(d \mathrm{Nu}_{\mathrm{i}} 0\right)$ e $\mathrm{k}_{\max }\left(d \mathrm{Nu}_{\mathrm{i}} \mathrm{k}_{\max }\right)$.

As $t r \mathrm{Nu}_{\mathrm{i}}-\mathrm{Pl}$ devem ser estimadas de duas formas:

a) $\operatorname{trN\mathrm {Nu}_{\mathrm {i}}} \mathrm{Pl}=\left(d \mathrm{Nu}_{\mathrm{i}} \mathrm{k}_{\max }-d \mathrm{Nu}_{\mathrm{i}} 0\right) / q d \mathrm{Nu}_{\mathrm{i}} \mathrm{k}_{\max }+\mathrm{Sb}_{\mathrm{c}}$

b) $\operatorname{tr} \mathrm{Nu}_{\mathrm{i}} \mathrm{Pl}=\left(d \mathrm{Nu}_{\mathrm{i}} \mathrm{k}_{\max }-d \mathrm{Nu}_{\mathrm{i}}-\mathrm{FS} 1 \mathrm{C}-\mathrm{Nu}_{\mathrm{i}}\right) / q d \mathrm{Nu}_{\mathrm{i}} \mathrm{k}_{\max }+\mathrm{Sb}_{\mathrm{c}}$

em que: $q d \mathrm{Nu}_{\mathrm{i}} \mathrm{k}_{\max }+\mathrm{Sb}_{\mathrm{c}}=q a \mathrm{Nu}_{\mathrm{i}} \mathrm{k}_{\max }+q d \mathrm{Nu}_{\mathrm{i}}-\mathrm{Sb}_{\mathrm{c}}$, sendo $q d \mathrm{Nu}_{\mathrm{i}}-\mathrm{Sb}_{\mathrm{c}}=q d \mathrm{Nu}_{\mathrm{i}}-\mathrm{Sb} / t r \mathrm{Nu}_{\mathrm{i}}-\mathrm{Ex}$.
Para g):

Calcular o requerimento dos nutrientes da planta: $r \mathrm{Nu}_{\mathrm{i}}=d \mathrm{Nu}_{\mathrm{i}} / t r \mathrm{Nu}_{\mathrm{i}}-\mathrm{Pl}$.

Para h):

Calcular o suprimento (quantidade disponibilizada) dos nutrientes pelo substrato:

$q d \mathrm{Nu}_{\mathrm{i}}-\mathrm{Sb}_{\mathrm{c}}=q d \mathrm{Nu}_{\mathrm{i}}-\mathrm{Sb} / t r \mathrm{Nu}_{\mathrm{i}}-\mathrm{Ex}$.

Para i):

Calcular as quantidades de nutrientes a serem suplementadas $\left(q s \mathrm{Nu}_{\mathrm{i}}-\mathrm{FS} 2\right)$ e os teores de nutrientes no FS2: $q s \mathrm{Nu}_{\mathrm{i}}-\mathrm{FS} 2=r \mathrm{Nu}_{\mathrm{i}}-q d \mathrm{Nu}_{\mathrm{i}}$.

Essa técnica experimental está começando a ser testada para produção de mudas de cafeeiro. Entretanto, para ilustrar a sua utilização, até a obtenção do fertilizante para complementar o suprimento de nutrientes (FS1), considerar-se-ia o cultivo de plantas de violeta.

Adquirir 50 vasos de violetas Kalise de flores rosa (Krs). Escolher pelo vigor e tamanho das plantas oito amostras de seis plantas. Separar as plantas em meristemas, folhas e inflorescências. Obter as $m \mathrm{MS}_{\mathrm{j}}$. Em média, a $m \mathrm{MS}_{\mathrm{j}}$ foi: meristema $0,24 \mathrm{~g} / \mathrm{planta}$; folhas $2,10 \mathrm{~g} /$ planta; e inflorescências $0,63 \mathrm{~g} / \mathrm{planta}$. Na MS de meristemas, folhas e inflorescências, foram determinados os teores totais de nutrientes. Os teores médios dos nutrientes por compartimento e os de formas disponíveis de nutrientes do substrato a serem usados, encontram-se no quadro 12.

Considerando a produção de MS e os teores de nutrientes i por compartimento j, foram calculados os conteúdos de nutrientes (Quadro 13).

O conteúdo total constitui a demanda de nutrientes pelas plantas $\left(d \mathrm{Nu}_{\mathrm{i}}\right)$. A demanda dividida pela taxa de recuperação de nutrientes pelas plantas obtém o requerimento de nutrientes $\left(r \mathrm{Nu}_{\mathrm{i}}\right)$ (Quadro 14). As $t r \mathrm{Nu}_{\mathrm{i}}-\mathrm{Pl}$ foram em parte utilizadas das obtidas por Matta (2012), mas, com modificações, especialmente, as de micronutrientes cátions.

O suprimento de nutrientes pelo substrato $\left(s \mathrm{Nu}_{\mathrm{i}}-\mathrm{Sb}_{\mathrm{c}}\right)$ foi calculado ao dividir os teores de nutrientes do substrato $\left(t d \mathrm{Nu}_{\mathrm{i}^{-}} \mathrm{Sb}\right)$ pelas taxas de recuperação dos extratores $\left(t r \mathrm{Nu}_{\mathrm{i}}\right.$-Ex). Pela diferença do requerimento menos o suprimento foram obtidas as quantidades de nutrientes que devem ser suplementadas pelo FS1 ( $q s \mathrm{Nu}_{\mathrm{i}}$-FS1) em solução, (Quadro 14), em 15 aplicações semanais de $25 \mathrm{~mL} / \mathrm{vaso}$.

Para produção de 2,97 g/planta da variedade Fiesta de flores rosa, é necessário adicionar 141,451 mg/vaso de N, em 15 aplicações semanais de FS1 em solução, ou seja, $157,168 \mathrm{mg} / \mathrm{vaso} / \mathrm{aplic}$ de FS1. O FS1 deve conter, em \% (m/m): $6,00 \mathrm{~N} ; 5,643 \mathrm{P} ; 4,949 \mathrm{~S}$; $15,217 \mathrm{~K} ; 4,589 \mathrm{Ca} ; 2,138 \mathrm{Mg} ; 0,0886 \mathrm{~B} ; 0,0017 \mathrm{Mo}$; 0,1353 Zn; 0,2248 Fe; 0,1438 Mn; e 0,0097 Cu (Quadro 14). Para preparar a solução de FS1, é necessário pesar $15,717 \mathrm{~g}$, dissolver e diluir até 2,5 L. Dessa solução, adicionar $25 \mathrm{~mL} / \mathrm{vaso} /$ aplic. 
Quadro 12. Teores de nutrientes por compartimento de plantas de violeta e teores de formas disponíveis de nutrientes do substrato

\begin{tabular}{|c|c|c|c|c|c|c|c|c|c|}
\hline Variável & Unid. & $\mathbf{N}$ & $\mathbf{P}$ & $\mathbf{S}$ & $\mathbf{K}$ & $\mathbf{C a}$ & B & $Z_{n}$ & $\mathbf{F e}$ \\
\hline & & & & $-\mathrm{g} \mathrm{kg}^{-1}$ & & & & $-\mathrm{mg} \mathrm{kg}^{-1}$ & \\
\hline$t t \mathrm{Nu}_{\mathrm{i}}-\mathrm{M}$ & & 33,100 & 6,500 & 1,800 & 39,400 & 7,200 & 35,500 & 357,300 & 93,700 \\
\hline$t t \mathrm{Nu}_{\mathrm{i}}-\mathrm{F}$ & & 40,900 & 6,900 & 2,500 & 48,100 & 20,100 & 49,700 & 92,100 & 210,500 \\
\hline$t t \mathrm{Nu}_{\mathrm{i}} \mathrm{-I}$ & & 48,300 & 22,800 & 2,100 & 57,300 & 13,500 & 55,300 & 81,200 & 112,300 \\
\hline$t d N u \mathrm{i}-\mathrm{Sb}$ & $\mathrm{mg} \mathrm{dm}{ }^{-3}$ & 50,500 & 13,500 & 24,800 & 148,600 & 88,000 & 0,110 & 1,800 & 4,500 \\
\hline
\end{tabular}

(1) $t$, $t d=$ teor total e teor disponível; $\mathrm{Nu}_{\mathrm{i}}=$ nutriente $\mathrm{i}$; e $\mathrm{M}, \mathrm{F}, \mathrm{I}$ e $\mathrm{Sb}=$ meristemas, folhas, inflorescências e substrato.

Quadro13. Conteúdos de nutrientes por compartimento e total de plantas de violeta

\begin{tabular}{|c|c|c|c|c|c|c|c|c|c|}
\hline Variável & Unid. & $\mathbf{N}$ & $\mathbf{P}$ & $\mathbf{S}$ & $\mathbf{K}$ & $\mathbf{C a}$ & B & $\mathrm{Zn}$ & $\mathbf{F e}$ \\
\hline$c \mathrm{Nu}_{\mathrm{i}}-\mathrm{M}$ & $\mathrm{mg} /$ planta & 7,944 & 1,560 & 0,432 & 9,456 & 1,728 & 0,009 & 0,086 & 0,023 \\
\hline$c \mathrm{Nu}_{\mathrm{i}}-\mathrm{F}$ & $\mathrm{mg} /$ planta & 85,890 & 14,490 & 5,250 & 101,010 & 42,210 & 0,104 & 0,193 & 0,442 \\
\hline$c \mathrm{Nu}_{\mathrm{i}}-\mathrm{I}$ & $\mathrm{mg} /$ planta & 30,429 & 14,364 & 1,323 & 36,099 & 8,505 & 0,035 & 0,051 & 0,071 \\
\hline$c \mathrm{Nu}_{\mathrm{i}}-\mathrm{T}$ & $\mathrm{mg} /$ planta & 124,263 & 30,414 & 7,005 & 146,565 & 52,443 & 0,148 & 0,330 & 0,535 \\
\hline
\end{tabular}

(1) $c$ = conteúdo; $\mathrm{Nu}_{\mathrm{i}}=$ nutriente $\mathrm{i} ; \mathrm{M}, \mathrm{F}, \mathrm{I}$ e T = meristemas, folhas, inflorescências e total.

Quadro 14. Demanda, requerimento, suprimento e teores de nutrientes do FS1

\begin{tabular}{|c|c|c|c|c|c|c|c|c|c|}
\hline Variável & Unid. & $\mathbf{N}$ & $\mathbf{P}$ & $\mathrm{S}$ & $\mathbf{K}$ & $\mathbf{C a}$ & B & $\mathrm{Zn}$ & $\mathrm{Fe}$ \\
\hline$d \mathrm{Nu}_{\mathrm{i}}$ & $\mathrm{mg} /$ planta & 124,263 & 30,414 & 7,005 & 146,565 & 52,443 & 0,148 & 0,330 & 0,535 \\
\hline $\operatorname{tr} \mathrm{Nu}_{\mathrm{i}}-\mathrm{Pl}$ & & 0,745 & 0,202 & 0,051 & 0,349 & 0,320 & 0,068 & 0,066 & 0,061 \\
\hline$r \mathrm{Nu}_{\mathrm{i}}$ & $\mathrm{mg} / \mathrm{vaso}$ & 166,701 & 150,889 & 137,843 & 420,509 & 164,096 & 2,184 & 5,016 & 8,735 \\
\hline$t d \mathrm{Nu}_{\mathrm{i}}-\mathrm{Sb}$ & $\mathrm{mg} / \mathrm{vaso}$ & 20,200 & 5,400 & 9,920 & 59,440 & 35,200 & 0,044 & 0,720 & 1,800 \\
\hline $\operatorname{tr} \mathrm{Nu}_{\mathrm{i}}-\mathrm{Ex}$ & & 0,800 & 0,302 & 0,469 & 0,962 & 0,630 & 0,456 & 0,394 & 0,524 \\
\hline$s \mathrm{Nu}_{\mathrm{i}}-\mathrm{Sb}_{\mathrm{c}}$ & $\mathrm{mg} / \mathrm{vaso}$ & 25,250 & 17,864 & 21,169 & 61,762 & 55,903 & 0,097 & 1,826 & 3,434 \\
\hline$q s \mathrm{Nu}_{\mathrm{i}}-\mathrm{FS} 1$ & $\mathrm{mg} / \mathrm{vaso}$ & 141,451 & 133,025 & 116,674 & 358,747 & 108,192 & 2,088 & 3,190 & 5,301 \\
\hline FS1 & $\%$ & 6,000 & 5,643 & 4,949 & 15,217 & 4,589 & 0,089 & 0,135 & 0,225 \\
\hline
\end{tabular}

(1) $d, r, t, t r, q d, s$ e $q s=$ demanda, requerimento, teor, taxa de recuperação, quantidade disponível (escala de teores), suprimento (escala doses) e quantidade suplementar; $\mathrm{Nu}_{\mathrm{i}}=$ nutriente i; e Pl, Ex, Sb e FS1 = plantas, extratores, substrato e Fertilizantesuprimento.

\section{AGRADECIMENTOS}

À Fundação de Amparo à Pesquisa do Estado de Minas Gerais (FAPEMIG), pelo suporte financeiro para pesquisas com plantas ornamentais; e ao Conselho Nacional de Desenvolvimento Científico e Tecnológico (CNPq), pelas bolsas concedidas.

\section{LITERATURA CITADA}

BARROS, N.F.; NOVAIS, R.F.; TEIXEIRA, J.L. \& FERNANDES FILHO, E.I. NUTRICALC 2.0 - Sistema para cálculo del balance nutricional y recomendación de fertilizantes para el cultivo de eucalipto. Bosque, 16:129-131, 1995.
DEUS, J.A.L. Sistema de recomendação de corretivos e fertilizantes para o meloeiro com base no balanço nutricional. Fortaleza, Universidade Federal do Ceará, 2012. 121p. (Dissertação de Mestrado)

MATTA, P.M. Recomendação de fertilizantes para violeta africana baseada na demanda nutricional da planta. Viçosa, MG, Universidade Federal de Viçosa, 2012. 74p. (Dissertação de Mestrado)

MERLE, M. La fertilisation en cultures fruitières exotiques. In: COLLOQUE TENU À ABIDJAN. Nutrition Minérale et Engrais. Abidjan, IFAC, IFCC, IRCT, IRCA, IRHO et ORSTOM, 1959. 43p. (Première Séance)

NOVAIS, R.F. \& SMYTH, T.J. Fósforo em solo e planta em condições tropicais. Viçosa, MG, Universidade Federal de Viçosa, 1999. 399p. 
PONTES, M.S. Parametrização do modelo 3-PG para Teca (Tectona grandis L. F.) e dos sistemas Ferti-UFV e NutriUFV para subsidiar o seu manejo nutricional. Viçosa, MG, Universidade Federal de Viçosa, 2011. 54p. (Dissertação de Mestrado)

SANTOS, A.F. Composição mineral do meio de cultura para crescimento in vitro de Cattleya walquiriana. Viçosa, MG, Universidade Federal de Viçosa, 2008. 24p. (Dissertação de Mestrado)
SANTOS, G.L.A.A. Adubação com macro e micronutrientes ajustada à demanda nutricional da violeta africana. Viçosa, MG, Universidade Federal de Viçosa, 2011. 30p. (Dissertação de Mestrado)

STAHRINGER, N.I. Sistema de recomendação de corretivos e fertilizantes para a cultura da laranjeira com base no balanço nutricional (FERTICALC - Laranja). Viçosa, MG, Universidade Federal de Viçosa, 2013. 120p. (Dissertação de Mestrado) 